\title{
A Hybrid Technique Based on Combining Fuzzy $K$ - means Clustering and Region Growing for Improving Gray Matter and White Matter Segmentation
}

\author{
Ashraf Afifi \\ Computer Engineering Dept., \\ Faculty of Computers and Information Technology \\ Taif University \\ Taif, KSA
}

\begin{abstract}
In this paper we present a hybrid approach based on combining fuzzy k-means clustering, seed region growing, and sensitivity and specificity algorithms to measure gray (GM) and white matter (WM) tissue. The proposed algorithm uses intensity and anatomic information for segmenting of MRIs into different tissue classes, especially GM and WM. It starts by partitioning the image into different clusters using fuzzy k-means clustering. The centers of these clusters are the input to the region growing (SRG) method for creating the closed regions. The outputs of SRG technique are fed to sensitivity and specificity algorithm to merge the similar regions in one segment. The proposed algorithm is applied to challenging applications: gray matter/white matter segmentation in magnetic resonance image (MRI) datasets. The experimental results show that the proposed technique produces accurate and stable results.
\end{abstract}

Keywords- Fuzzy clustering; seed region growing; performance measure; MRI brain database; sensitivity and specificity.

\section{INTRODUCTION}

Medical imaging includes conventional projection radiography, computed topography (CT), magnetic resonance imaging (MRI) and ultrasound. MRI has several advantages over other imaging techniques enabling it to provide 3D data with high contrasts between soft tissues. However, the amount of data is far too much for manual analysis/interpretation, and this has been one of the biggest obstacles in the effective use of MRI.

The segmentation of region is an important first step for variety of image related applications and visualization tasks. Also, segmentation of medical images is important since it provides assistance for medical doctors to find out the diseases inside the body without the surgery procedure, to reduce the image reading time, to find the location of a lesion and to determine an estimate the probability of a disease. Segmentation of brain MRIs into different tissue classes, especially gray matter (GM), and white matter (WM), is an important task. Brain MRIs have low contrast between some different tissues. The problem of MRIs is the low contrast between tissues.

The measurement of GM of MRI has become an important tool for determining the multiple sclerosis (MS) patient monitoring. In the past, MS was considered primarily a white matter (WM) disease visible by macroscopic examination of the tissue and on MRI. Histological studies of MS brain tissue have provided that MS lesions are also located in the gray matter and that these GM lesions make up a substantial proportion of overall tissue damage due to MS [1]. To measure the changes over time in GM volumes, accurate segmentation methods must be used. A variety of different approaches to brain tissue segmentation has been described in the literature [2-4]. Few algorithms rely solely on image intensity, [2] because these approaches are overly sensitive to image artifacts such as radio frequency inhomogeneity, and aliasing, and cannot adequately account for overlapping intensity distributions across structures. Therefore, to improve segmentation accuracy, most tissue segmentation algorithms combine intensity information with other techniques, such as the use of a priori anatomic information [3, 4] or edge information through deformable contours. The use of multiple images has significant advantages over a single image because the different contrasts can be enhanced between tissues. For example, fluid attenuated inversion recovery (FLAIR) images have desirable contrast between MS lesions and the normalappearing brain tissue and can be combined with other images to obtain gray/white matter segmentation.

In other hand, several algorithms have been proposed such as: fuzzy k-means [7], c-means (FCM) [5], and adaptive fuzzy c-means combined with neutrosophic set to improve MRI segmentation. These algorithms, such as the segmentation tool in SPM, [6] and FAST in FSL [7], have been implemented for general use, and therefore, are not necessarily optimized for specific pulse sequences or for application to images from patients with a specific disease.

These methods are also prone to classification errors due to partial volume effects between MS lesions and normal tissue. Furthermore, for retrospective image analysis, where image data may not have been acquired using optimal sequences for use with one of the widely available segmentation tools, a customized segmentation method may be required to obtain the most accurate results.

In this paper, we present an approach based on combining fuzzy c-mean clustering, seed region growing, and sensitivity and specificity algorithm to determine GM and WM tissues in brain MRIs. This approach begins by partitioning the given 
image into several regions. The seed region growing method is applied to the image using the centers of these regions as initial seeds (if this center is not in image, a quite neighbor point to this center is selected as initial seed). Then the sensitivity and specificity is used to perform a suitable merging which produces the final segmentation. The proposed method is evaluated and compared with the existing methods by applying them on simulated volumetric MRI datasets.

The rest of the paper is organized as follows. The MRI segmentation problem is discussed in section 2 . The proposed method is described in section 3. In Section 4, the experimental results are presented. Our conclusion is presented in section5.

\section{THE MRI SEGMENTATION PROBLEM}

The basic idea of image segmentation can be described as follows. Suppose that $X=\{x 1, x 2, \ldots, x n\}$ is a given set of data and $\mathrm{P}$ is a uniformity set of predicates. We aim to obtain a partition of the data into disjoint nonempty groups $X=\{v 1$, $\mathrm{v} 2, \ldots, \mathrm{vk}$ \} subject to the following conditions:

$$
\begin{aligned}
& \mathrm{Y}_{i=1}^{k} v_{i}=X \\
& v_{i} \mathrm{I} \quad v_{j}=\phi, \mathrm{i} \neq \mathrm{j} \\
& P\left(v_{i}\right)=T R U E, i=1,2, . ., k \\
& P\left(v_{i} \mathrm{Y} v_{j}\right)=F A L S E, i=j
\end{aligned}
$$

The first condition ensures that every data value must be assigned to a group, while the second condition ensures that a data value can be assigned to only one group. The third and fourth conditions imply that every data value in one group must satisfy the uniformity predicate while data values from two different groups must fail the uniformity criterion.

Our study is related to 3D-model from MRI and simulated brain database of McGill University [14]. MRI has several advantages over other imaging techniques enabling it to provide 3-dimensional data with high contrast between soft tissues. However, the amount of data is far too much for manual analysis/interpretation, and this has been one of the biggest obstacles in the effective use of MRI. Segmentation of MR images into different tissue classes, especially gray matter (GM), white matter (WM) and cerebrospinal fluid (CSF), is an important task.

\section{THE PROPOSED ALGORITHM DESCRIPTION}

The objective of image segmentation is to divide an image into meaningful regions. Errors made at this stage would affect all higher level activities. In an ideally segmented image, each region should be homogeneous with respect to some criteria such as gray level, color or texture, and adjacent regions should have significantly different characteristics or feature. In MRI segmentation, accurate segmentation of white matter (WM) and gray matter (GM) is critically important in understanding structural changes associated with central nervous system diseases such as multiple sclerosis and Alzheimer's disease, and also the normal aging process [8]. Measures of change in WM and GM volume are suggested to be important indicators of atrophy or disease progression. In many situations, it is not easy to determine if a voxel should belong to WM or GM. This is because the features used to determine homogeneity may not have sharp transitions at region boundaries. To alleviate this situation, we propose an approach based on fuzzy set and seed region growing concepts into the segmentation process. If the memberships are taken into account while computing properties of regions, we obtain more accurate estimates of region properties. Our segmentation strategy will use the fuzzy k-means (FKM) for finding optimum seed as a presegmentation tool, seed region growing algorithm will operate on this seed to obtain close regions, and then refine the results using the performance measure. We use sensitivity and specificity [9] as performance measure to compare the performance of various outputs of the seed region growing method. The proposed algorithm is described in Fig.(1). The advantage of the proposed approach is that it combines the advantages of both methods: the FKM pre-segmentation is rough but quick, and the seed region growing needs only the initial seed point to produce the final, fast, highly accurate and smooth segmentation.

The proposed algorithm consists of three procedures:

- $\quad$ FKM algorithm for finding optimum seed;

- Seed region growing to isolate suitable regions;

- Performance measure procedure for merging regions and extracting the final segmentation.

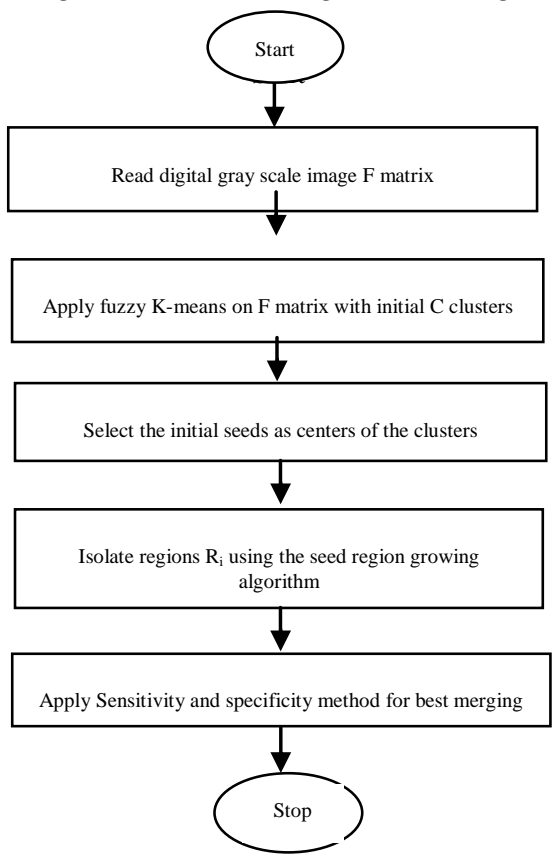

Figure1. The steps of the proposed algorithm

\section{A. The fuzzy K-means clustering}

Fuzzy K-means clustering (FKM) algorithm partitions data points into k clusters $s_{1}(I=1,2, \ldots ., k)$ and clusters $S_{1}$ are associated with representatives (cluster center) $C_{1}$ [7]. The relationship between a data point and cluster representive is fuzzy. That is, a membership $u_{i j} \in[1,0]$ is used to represent the degree of belongingness of data point $X_{i}$ and cluster center $C_{j}$. 
Denote the set of data points as $S=\left\{X_{i}\right\}$. The FKM algorithm is based on minimizing the following distortion:

$$
J=\sum_{i=1}^{k} \sum_{j=1}^{N} u_{i j}^{m} d_{i j}
$$

With respect to the cluster representatives $C_{j}$ and memberships $u_{i j}$, where $N$ is the number of data points; $m$ is the fuzzifier parameter; $k$ is the number of clusters; and $d_{i j}$ is the squared Euclidean distance between

data points $X_{i}$ and cluster representative $C_{j}$. It is noted that $u_{i j}$ should satisfy the following constraint:

$$
\sum_{i=1}^{k} u_{i j}=1, \quad \forall j=1, \ldots \ldots, N .
$$

The major process of FKM is mapping a given set of representive vectors into an improved one through partitioning data points. It begins with a set of initial cluster centers and repeats this mapping process until a stopping criterion is satisfied. It is supposed that no two clusters have the same cluster representative. In the case that two cluster centers coincide, a cluster center should be perturbed to avoid coincidence in the iterative process. If $d_{i j}<\eta$, then $u_{i j}=1$ and $u_{i j}=0$ for $i \neq j$, where $\eta$ is a very small positive number. The fuzzy k-means clustering algorithm is now presented as follows.

1) Input a set of initial cluster centers $S C_{o}=\left\{C_{j}(0)\right\}$ and the value $\varepsilon$. Set $P=1$.

2) Given the set of cluster centers $S C_{p}$, compute $d_{i j}$ for $i=1$ to $N$ and $j=1$ to $k$.

Update memberships $u_{i j}$ using the following equation:

$$
u_{i j}=\left(\left(d_{i j}\right)^{\frac{1}{m-1}} \sum_{i=1}^{k}\left(\frac{1}{d_{i j}}\right)^{\frac{1}{m-1}}\right)^{-1}
$$

If $d_{i j}<\eta$, set $u_{i j}=1$, where $\eta$ is a very small

positive number.

3) Compute the center for each cluster using Eq.(4) to obtain a new set of cluster representatives $S C_{p+1}$,

$$
c_{j(p)}=\frac{\sum_{i=1}^{N} u_{i j}^{m} x_{i}}{\sum_{i=1}^{N} u_{i j}^{m}}
$$

If

$\begin{array}{ll}\left\|C_{j}(P)-C_{j}(p-1)\right\|<\varepsilon \text { for } j=1 \text { to } k, & \text { where } \\ \text { then } & \text { stop, } \\ \left\|C_{j}(P)-C_{j}(p-1)\right\|<\varepsilon \text { for } j=1 \text { to } k, & \varepsilon>0\end{array}$

is a very small positive number.
Otherwise set $p+1 \rightarrow p$ and go to step 2 .

\section{B. Seed region growing}

In this section, we select the center of the cluster or the nearest point to this cluster as an initial seed of region growing algorithm. The seed position (pixel_x, pixel_y) can be grown by merging neighboring pixels whose properties are most similar to the premerged region. The neighbors can be chosen based on either their distance from the seed point or the statistical properties of the neighborhood. Then each of the 4 or 8 neighbours of that pixel are visited to determine if they belong to the same region. This growing expands further by visiting the neighbours of each of these 4 or 8 neighbor pixels. This recursive process continues until either some termination crierion is met or all pixels in the image are examined. The result is a set of connected pixels determined to be located within the region of interest. The algorithm used for this task can be stated in two steps [10]:

\section{Step1: Gradient based homogeneity criteria}

Success of region grow algorithm depends on the initial seed selection and criteria used to terminate the recursive region grow. Hence choosing appropriate criteria is the key in extracting the desired regions. In general, these criteria include region homogeneity, object contrast with respect to background, strength of the region boundary, size, and conformity to desired texture features like texture, shape, and color.

We used criteria mainly based on region homogeneity and region aggregation using intensity values and their gradient direction and magnitude. This criterion is characterized by a cost function which exploits certain features of images around the seed [11]. These cost functions are verified for their match with the specified conditions of homogeneity criteria by comparing their values. If there is a match then pixel under consideration is added to the growing region otherwise excluded from consideration.

Gradient based cost functions used in our implementation are defined below.

$$
G_{n}=\sqrt{G_{x}^{2}+G_{y}^{2}} / k G_{\max }
$$

Such that $0<G_{n}<1$

Where $G_{x}$ is the horizontal gradient component, $G_{y}$ is the vertical gradient component; $\mathrm{k}$ is the constant parameter which controls the region grow, and $\mathrm{G}_{\max }$ is the largest gradient magnitude present in the image.

$$
\begin{gathered}
G_{m}=G_{\max }-G(x, y) / G_{\max }-G_{\min } \\
\text { Such that } 0<G_{m}<1
\end{gathered}
$$

Where $G(x, y)$ is the gradient magnitude at pixel under consideration and $G_{\min }$ is the minimum gradient present in the image.

\section{Step2: Stack based seeded region growing algorithm}

We have implemented the 2D seeded region grow algorithm using stack data structure. Since, the stack is simple 
to implement and efficient in the data access, we used stack to traverse the neighborhood pixels around the seed location. In our implementation we considered 4-neighbours while growing the region as shown in Fig.(2). Similar pseudo code for our implementation is as follows:

Initialize the stack:

For each seed location

Push seed location to stack

While (stack not empty)

Pop location

Mark location as region

Mark location as visited node

If homogeneity criteria matches for location's left neighbor pixel

$$
\begin{aligned}
& \text { If left neighbor is not visited } \\
& \text { Push left neighbor to stack }
\end{aligned}
$$

If homogeneity criteria matches for location's To $\mathrm{p}$ neighbor pixel

If top neighbor is not visited

Push top neighbor to stack

If homogeneity criteria matches for location's bottom neighbor pixel

If bottom neighbor is not visited

Push bottom neighbor to stack

End

\begin{tabular}{|l|l|l|}
\hline $\mathrm{x}-1, \mathrm{y}-1$ & $\mathrm{x}, \mathrm{y}-1$ & $\mathrm{x}+1, \mathrm{y}-1$ \\
\hline $\mathrm{x}-1, \mathrm{y}$ & $\mathrm{x}, \mathrm{y}$ & $\mathrm{x}+1, \mathrm{y}$ \\
\hline $\mathrm{x}-1, \mathrm{y}+1$ & $\mathrm{x}, \mathrm{y}+1$ & $\mathrm{x}+1, \mathrm{y}+1$ \\
\hline
\end{tabular}

Figure 2. Four neighbors considered for region grow

Similar concept is extended for segmentation of 3D data set using region growing method as shown in Fig. (2). In 3D segmentation, 6 neighbors are considered during segmentation. Two additional pixels along $\mathrm{z}$-axis from 2 adjacent slices are considered along with 4 neighbors.

\section{Performance measures}

To compare the performance of various outputs of seed region growing technique, several methods such as: Jaccard similarity coefficient [12], Dice similarity coefficient [13], sensitivity and specificity [10] are used. In this section, we use sensitivity (SENS) and specificity (SPEC) method which almost gives good stable results [9]. Below, let consider the sensitivity and specificity measure. Sensitivity and specificity are statistical measures of the performance of a binary classification test, commonly used in medical studies. Sensitivity measures the proportion of the automatically segmented region $R_{l}$ pixels that are correctly identified as such. Specificity measures the proportion of the correspondent region of the manually segmented image $R_{2}$ pixels that are correctly identified. Given the following definitions:

$\boldsymbol{T P}$ is true positive, $R_{I}$ pixels that are correctly classified as interest $R_{l}$.

$\boldsymbol{F P}$ is false positive, $R_{2}$ pixels that are incorrectly identified as interest $R_{l}$.

$\boldsymbol{T N}$ is true negative, $R_{2}$ pixels that are correctly identified as $R_{2}$.

$\boldsymbol{F N}$ is false negative, $R_{l}$ pixels that are incorrectly identified as $\mathrm{R}_{2}$.

We compute different coefficients reflecting how well two

segmented regions match. The Sensitivity and specificity is formulated as follows [13]:

$$
\begin{aligned}
& S E N S=\frac{T P}{T P+T N} \\
& S P E C=\frac{T N}{F P+T N}
\end{aligned}
$$

A SENS of 1.0 represents perfect overlap. In this case two regions can be merged into one segment. Whereas an index of 0.0 represents no overlap.

Algorithm 3: Sensitivity and specificity measure similarity

$$
\text { Input } R_{i}, i=1,2,3, \ldots . ., k
$$

For $\mathrm{i}=1$ to $\mathrm{k}$

$$
\text { For } \mathrm{j}=2 \text { to } \mathrm{k}
$$

$$
\begin{aligned}
& \text { Compute } \operatorname{SENS}\left(\mathrm{R}_{\mathrm{i}}, \mathrm{R}_{\mathrm{j}}\right) \\
& \text { Compute } \operatorname{SPEC}\left(\mathrm{R}_{\mathrm{i}}, \mathrm{R}_{\mathrm{j}}\right)
\end{aligned}
$$

$$
\text { If ABS }(\mathrm{SENS}-\mathrm{SPEC})<0.5 \text { then } R_{i}=\left(\boldsymbol{R}_{i} \cup \boldsymbol{R}_{j}\right)
$$

End If

\section{End For}

End For

End;

Numerical results

For example, if one has a 7x7 discrete image $\mathrm{F}$ on the square grid (see Figure 3(a)). We can apply our algorithm as the following:

Step1:According to the fuzzy k-means clustering algorithm, we can divide the image $\mathrm{F}$ into six clusters with centers $(1,32,53,29,77,2)$ as shown in figure $(3(b, c, d, e, f, g))$.

\begin{tabular}{|c|c|c|c|c|c|c|}
\hline 1 & 1 & 2 & 50 & 30 & 29 & 29 \\
\hline 2 & 1 & 2 & 55 & 31 & 32 & 30 \\
\hline 1 & 1 & 1 & 0 & 30 & 31 & 29 \\
\hline 2 & 1 & 1 & 0 & 0 & 30 & 31 \\
\hline 3 & 2 & 1 & 77 & 33 & 32 & 28 \\
\hline 1 & 1 & 1 & 0 & 31 & 30 & 29 \\
\hline 1 & 0 & 1 & 0 & 28 & 33 & 32 \\
\hline \multicolumn{7}{|c|}{ (a) }
\end{tabular}




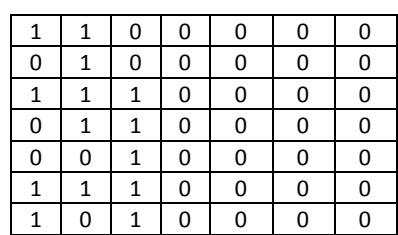

(b)

\begin{tabular}{|c|c|c|c|c|c|c|}
\hline 0 & 0 & 0 & 50 & 0 & 0 & 0 \\
\hline 0 & 0 & 0 & 55 & 0 & 0 & 0 \\
\hline 0 & 0 & 0 & 0 & 0 & 0 & 0 \\
\hline 0 & 0 & 0 & 0 & 0 & 0 & 0 \\
\hline 0 & 0 & 0 & 0 & 0 & 0 & 0 \\
\hline 0 & 0 & 0 & 0 & 0 & 0 & 0 \\
\hline 0 & 0 & 0 & 0 & 0 & 0 & 0 \\
\hline
\end{tabular}

(d)

\begin{tabular}{|l|l|l|l|l|l|l|}
\hline 0 & 0 & 0 & 0 & 0 & 0 & 0 \\
\hline 0 & 0 & 0 & 0 & 0 & 0 & 0 \\
\hline 0 & 0 & 0 & 0 & 0 & 0 & 0 \\
\hline 0 & 0 & 0 & 0 & 0 & 0 & 0 \\
\hline 0 & 0 & 0 & 77 & 0 & 0 & 0 \\
\hline 0 & 0 & 0 & 0 & 0 & 0 & 0 \\
\hline 0 & 0 & 0 & 0 & 0 & 0 & 0 \\
\hline
\end{tabular}

(f)

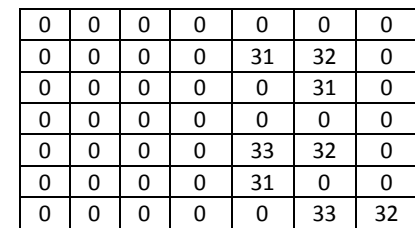

\begin{tabular}{|c|c|c|c|c|c|c|}
\hline 0 & 0 & 0 & 0 & 30 & 29 & 29 \\
\hline 0 & 0 & 0 & 0 & 0 & 0 & 30 \\
\hline 0 & 0 & 0 & 0 & 30 & 0 & 29 \\
\hline 0 & 0 & 0 & 0 & 0 & 30 & 0 \\
\hline 0 & 0 & 0 & 0 & 0 & 0 & 28 \\
\hline 0 & 0 & 0 & 0 & 0 & 30 & 29 \\
\hline 0 & 0 & 0 & 0 & 28 & 0 & 0 \\
\hline
\end{tabular}

(e)

\begin{tabular}{|l|l|l|l|l|l|l|}
\hline 0 & 0 & 2 & 0 & 0 & 0 & 0 \\
\hline 2 & 0 & 2 & 0 & 0 & 0 & 0 \\
\hline 0 & 0 & 0 & 0 & 0 & 0 & 0 \\
\hline 2 & 0 & 0 & 0 & 0 & 0 & 0 \\
\hline 3 & 2 & 0 & 0 & 0 & 0 & 0 \\
\hline 0 & 0 & 0 & 0 & 0 & 0 & 0 \\
\hline 0 & 0 & 0 & 0 & 0 & 0 & 0 \\
\hline \multicolumn{7}{|c|}{$(\mathrm{g})$}
\end{tabular}

Figure 3.The fuzzy k-means clustering, (a) Original image, and (c-g) clusters

Step2:according to seed region growing algorithm2, we can obtain six regions $\mathrm{R}_{1}, \mathrm{R}_{2}, \mathrm{R}_{3}, \mathrm{R}_{4}, \mathrm{R}_{5}$, and $\mathrm{R}_{6}$ as shown in figure (4(a,b,c,d,e,f)).

\begin{tabular}{|l|l|l|l|l|l|l|}
\hline 1 & 1 & 2 & 0 & 0 & 0 & 0 \\
\hline 2 & 1 & 2 & 0 & 0 & 0 & 0 \\
\hline 1 & 1 & 1 & 0 & 0 & 0 & 0 \\
\hline 2 & 1 & 1 & 0 & 0 & 0 & 0 \\
\hline 3 & 2 & 1 & 0 & 0 & 0 & 0 \\
\hline 1 & 1 & 1 & 0 & 0 & 0 & 0 \\
\hline 1 & 0 & 1 & 0 & 0 & 0 & 0 \\
\hline
\end{tabular}

\begin{tabular}{|l|l|l|l|l|l|l|}
\hline 0 & 0 & 0 & 50 & 0 & 0 & 0 \\
\hline 0 & 0 & 0 & 55 & 0 & 0 & 0 \\
\hline 0 & 0 & 0 & 0 & 0 & 0 & 0 \\
\hline 0 & 0 & 0 & 0 & 0 & 0 & 0 \\
\hline 0 & 0 & 0 & 0 & 0 & 0 & 0 \\
\hline 0 & 0 & 0 & 0 & 0 & 0 & 0 \\
\hline 0 & 0 & 0 & 0 & 0 & 0 & 0 \\
\hline \multicolumn{7}{|c|}{ (c) }
\end{tabular}

\begin{tabular}{|c|c|c|c|c|c|c|}
\hline 0 & 0 & 0 & 0 & 0 & 0 & 0 \\
\hline 0 & 0 & 0 & 0 & 0 & 0 & 0 \\
\hline 0 & 0 & 0 & 0 & 0 & 0 & 0 \\
\hline 0 & 0 & 0 & 0 & 0 & 0 & 0 \\
\hline 0 & 0 & 0 & 77 & 0 & 0 & 0 \\
\hline 0 & 0 & 0 & 0 & 0 & 0 & 0 \\
\hline 0 & 0 & 0 & 0 & 0 & 0 & 0 \\
\hline
\end{tabular}

(e)

\begin{tabular}{|c|c|c|c|c|c|c|}
\hline 0 & 0 & 0 & 0 & 30 & 29 & 29 \\
\hline 0 & 0 & 0 & 0 & 31 & 32 & 30 \\
\hline 0 & 0 & 0 & 0 & 30 & 31 & 29 \\
\hline 0 & 0 & 0 & 0 & 0 & 30 & 31 \\
\hline 0 & 0 & 0 & 0 & 33 & 32 & 28 \\
\hline 0 & 0 & 0 & 0 & 31 & 30 & 29 \\
\hline 0 & 0 & 0 & 0 & 28 & 33 & 32 \\
\hline
\end{tabular}

(b) (d)

\begin{tabular}{|l|l|l|l|l|l|l|}
\hline 1 & 1 & 2 & 0 & 0 & 0 & 0 \\
\hline 2 & 1 & 2 & 0 & 0 & 0 & 0 \\
\hline 1 & 1 & 1 & 0 & 0 & 0 & 0 \\
\hline 2 & 1 & 1 & 0 & 0 & 0 & 0 \\
\hline 3 & 2 & 1 & 0 & 0 & 0 & 0 \\
\hline 1 & 1 & 1 & 0 & 0 & 0 & 0 \\
\hline 1 & 0 & 1 & 0 & 0 & 0 & 0 \\
\hline \multicolumn{7}{|c|}{ (f) }
\end{tabular}

Figure 4.The seed region growing, (a-f) regions

Step3: According to sensitivity and specificity measure algorithm3, we compute SENS and SPEC measure between regions (a-f) as shown in figure (5(a-f)).

From the previous calculation, we note that SENS and SPEC of regions $(1,6)$ and regions $(2,4)$ are high. Therefore, the regions $\left(R_{l}, R_{6}\right)$ and $\left(R_{2}, R_{4}\right)$ can be merged according sensitivity and specificity measure as shown in figure $(6(a, b, c, d))$.

\begin{tabular}{|c|c|c|}
\hline $\mathrm{R}$ & $\mathrm{SENS}$ & $\mathrm{SPEC}$ \\
\hline 1,2 & 0 & 0.5 \\
\hline 1,3 & 0 & 0.57 \\
\hline 1,4 & 0 & 0.31 \\
\hline 1,5 & 0 & 0.58 \\
\hline 1,6 & 1 & 1 \\
\hline
\end{tabular}

(a)

\begin{tabular}{|c|c|c|}
\hline$R$ & SENS & SPEC \\
\hline 4,1 & 0 & 0.31 \\
\hline 4,2 & 1 & 1 \\
\hline 4,3 & 0 & 0.93 \\
\hline 4,5 & 0 & 0.51 \\
\hline 4,6 & 0 & 0.25 \\
\hline
\end{tabular}

(d)

\begin{tabular}{|c|c|c|}
\hline$R$ & SENS & SPEC \\
\hline 2,1 & 0 & 0.5 \\
\hline 2,3 & 0 & 0.574 \\
\hline 2,4 & 1 & 1 \\
\hline 2,5 & 0 & 0.58 \\
\hline 2,6 & 0 & 0.31 \\
\hline \multicolumn{3}{|c|}{ (b) } \\
\hline
\end{tabular}

\begin{tabular}{|c|c|c|}
\hline$R$ & SENS & SPEC \\
\hline 5,1 & 0 & 0.58 \\
\hline 5,2 & 0 & 0.58 \\
\hline 5,3 & 0 & 0.92 \\
\hline 5,4 & 0 & 0.51 \\
\hline 5,6 & 0 & 0.966 \\
\hline
\end{tabular}

(e)

\begin{tabular}{|c|c|c|}
\hline$R$ & SENS & SPEC \\
\hline 3,1 & 0 & 0.57 \\
\hline 3,2 & 0 & 0.574 \\
\hline 3,4 & 0 & 0.93 \\
\hline 3,5 & 0 & 0.92 \\
\hline 3,6 & 0 & 0.93 \\
\hline
\end{tabular}

(c)

\begin{tabular}{|c|c|c|}
\hline$R$ & SENS & SPEC \\
\hline 6,1 & 1 & 1 \\
\hline 6,2 & 0 & 0.31 \\
\hline 6,3 & 0 & 0.93 \\
\hline 6,4 & 0 & 0.25 \\
\hline 6,5 & 0 & 0.966 \\
\hline
\end{tabular}

(f)
Figure 5.The sensitivity and specificity measure between regions, (a-f)

\begin{tabular}{|l|l|l|l|l|l|l|}
\hline 1 & 1 & 2 & 0 & 0 & 0 & 0 \\
\hline 2 & 1 & 2 & 0 & 0 & 0 & 0 \\
\hline 1 & 1 & 1 & 0 & 0 & 0 & 0 \\
\hline 2 & 1 & 1 & 0 & 0 & 0 & 0 \\
\hline 3 & 2 & 1 & 0 & 0 & 0 & 0 \\
\hline 1 & 1 & 1 & 0 & 0 & 0 & 0 \\
\hline 1 & 0 & 1 & 0 & 0 & 0 & 0 \\
\hline
\end{tabular}

\begin{tabular}{|l|l|l|l|l|l|l|}
\hline 0 & 0 & 0 & 50 & 0 & 0 & 0 \\
\hline 0 & 0 & 0 & 55 & 0 & 0 & 0 \\
\hline 0 & 0 & 0 & 0 & 0 & 0 & 0 \\
\hline 0 & 0 & 0 & 0 & 0 & 0 & 0 \\
\hline 0 & 0 & 0 & 0 & 0 & 0 & 0 \\
\hline 0 & 0 & 0 & 0 & 0 & 0 & 0 \\
\hline 0 & 0 & 0 & 0 & 0 & 0 & 0 \\
\hline
\end{tabular}

(c)

\begin{tabular}{|c|c|c|c|c|c|c|}
\hline 0 & 0 & 0 & 0 & 30 & 29 & 29 \\
\hline 0 & 0 & 0 & 0 & 31 & 32 & 30 \\
\hline 0 & 0 & 0 & 0 & 30 & 31 & 29 \\
\hline 0 & 0 & 0 & 0 & 0 & 30 & 31 \\
\hline 0 & 0 & 0 & 0 & 33 & 32 & 28 \\
\hline 0 & 0 & 0 & 0 & 31 & 30 & 29 \\
\hline 0 & 0 & 0 & 0 & 28 & 33 & 32 \\
\hline
\end{tabular}

(b)

\begin{tabular}{|l|l|l|l|l|l|l|}
\hline 0 & 0 & 0 & 0 & 0 & 0 & 0 \\
\hline 0 & 0 & 0 & 0 & 0 & 0 & 0 \\
\hline 0 & 0 & 0 & 0 & 0 & 0 & 0 \\
\hline 0 & 0 & 0 & 0 & 0 & 0 & 0 \\
\hline 0 & 0 & 0 & 77 & 0 & 0 & 0 \\
\hline 0 & 0 & 0 & 0 & 0 & 0 & 0 \\
\hline 0 & 0 & 0 & 0 & 0 & 0 & 0 \\
\hline
\end{tabular}

(d)
Figure 6 . The merged regions after sensitivity and specificity measuring

\section{EXPERIMENTAL RESULTS}

The experiments were performed with several data sets using MATLAB. We used a high-resolution T1-weighted MR phantom with slice thickness of $1 \mathrm{~mm}$, different noise and obtained from the classical simulated brain database of McGill University Brain Web [14] (see Fig.(7)).

The advantages of using digital phantoms rather than real image data for validating segmentation methods include prior knowledge of the true tissue types and control over image parameters such as modality, slice thickness, noise, and intensity inhomogeneities.

The quality of the segmentation algorithm is of vital importance to the segmentation process.

The comparison score $\mathrm{S}$ for each algorithm can be found in Zanaty et al.[15-17], and defined as:

$$
S=\left|\frac{A \cap A_{r e f}}{A \cup A_{r e f}}\right|
$$

Where $A$ represents the set of pixels belonging to a class as found by a particular method and $A_{\text {ref }}$ represents the reference cluster pixels. 


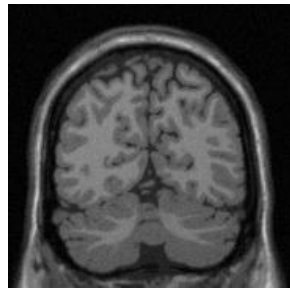

Figure. 7 Test image original slice\#62.

\section{A. Experiment on MRIs}

The original image size is $129 \times 129$ pixels, as shown in Fig. 7 obtained from the classical simulated brain. We apply our technique to segment images generated at various noise levels $(0 \%, 1 \%, 3 \%, 5 \%, 7 \%$, and $9 \%)$.We generate various inhomogeneities and boundary weakness by controlling noise and RF levels (0\% and 20\%) respectively.

Table I shows the score $S$ of WM using our technique at various noise and RF levels. These results show that our algorithm is very robust to noise and intensity; homogeneities and inhomogeneities. The best $S$ is achieved for low noise and low RF, for which values of $S$ are higher than 0.97 .

TABLE I THE SCORE $S$ OF WM

\begin{tabular}{|c|c|c|}
\hline Noise/RF & 0 & $20 \%$ \\
\hline $0 \%$ & 0.97 & 0.95 \\
\hline $1 \%$ & 0.96 & 0.94 \\
\hline $3 \%$ & 0.94 & 0.93 \\
\hline $5 \%$ & 0.90 & 0.92 \\
\hline $7 \%$ & 0.88 & 0.84 \\
\hline $9 \%$ & 0.85 & 0.80 \\
\hline
\end{tabular}

\section{B. Comparative results}

In this section, we compare the performance of our technique with two recent methods: Del-Fresno et al. [19] and $\mathrm{Yu}$ et al. [20] techniques which gave good results in brain segmentation. The segmentation results of these algorithms are presented in Figs.(6a), (6b), and (6c) respectively. The performance of each segmentation method on this dataset is reported in Table 2.

Table II shows the scort $\mathrm{S}$ of WM using different techniques for the Brain data. In this Table, we compare between proposed method, Del-Fresno et al. [18] and Yu et al. [19] techniques. In particular, although the segmentation quality logically deteriorates in the presence of noise $(0 \%$ and $6 \%$ ) and variations in intensity, the robustness of the present technique is highly satisfactory compared with the results of other segmentation techniques [18,19].

TABLE II THE SCORE FOR WM USING THE BRAIN WEB [14].

\begin{tabular}{llllll}
\hline Noise & $3 \%$ & & & $6 \%$ & \\
\cline { 2 - 3 } \cline { 5 - 6 } RF & $0 \%$ & $20 \%$ & & $0 \%$ & $20 \%$ \\
Proposed method & 0.94 & 0.93 & & 0.92 & 0.85 \\
Del-Fresno et al.[3] & 0.94 & 0.89 & & 0.91 & 0.84 \\
Yu et al.[6] & 0.90 & 0.90 & & 0.88 & 0.83 \\
\hline
\end{tabular}

\section{CONCLUSION}

In this paper, we have presented an approach for medical image segmentation, which integrates three existing methods: fuzzy $k$-means clustering, seed region growing, and sensitivity and specificity algorithms. The first two methods have a common advantage: they have no constraints or hypothesis on topology, which may change during convergence. The third method is used to merge similar regions. An initial partitioning of the image into primitive regions has been performed by applying a fuzzy clustering on the image. This initial partition is the input to a computationally efficient seed region that produces the suitable segmentation. The sensitivity and specificity algorithm is used to perform a suitable merging of regions which produces the final segmentation. It is observed that the proposed method has shown higher robustness in discrimination of regions because of the low signal/noise ratio characterizing most of medical images data.

By comparing the proposed methods with Del-Fresno et al. [18] and Yu et al. [19] methods, it is clear that the proposed algorithm can estimate the correct tissues WM and GM much more accurately than the established algorithms. Although, the accuracy of WM and GM clusters are varied according to noise factor, but we have shown that the proposed method gives better accuracy than Del-Fresno et al. [18] and Yu et al. [19] techniques with high noise level.

Future research in MRI segmentation should strive toward improving the computation speed of the segmentation algorithms, while reducing the amount of manual interactions needed. This is particularly important as MR imaging is becoming a routine diagnostic procedure in clinical practice. It is also important that any practical segmentation algorithm should deal with $3 \mathrm{D}$ volume segmentation instead of $2 \mathrm{D}$ slice by slice segmentation, since MRI data is $3 \mathrm{D}$ in nature.

\section{REFERENCES}

[1] Peterson, J.W., Bo, L., Mork, S., et al., " Transected neuritis, apoptotic neurons, and Reduced inflammation in cortical multiple sclerosis lesions", Ann. Neurol. 50, 389-400, 2001.

[2] De Stefano, N., Matthews, P.M., Filippi, M., Agosta, F., De Luca, M., Bartolozzi, M.L., Guidi,L., Ghezzi, A., Montanari, E., Cifelli, A., Federico, A., Smith, S.M.," Evidence of early cortical atrophy in MS: relevance to white matter changes and disability", Neurology 60 (7), 1157-1162, 2003.

[3] Schnack, H.G., Hulshoff Pol, H.E., Baaré, W.F.C., Staal, W.G., Viergever, M.A., Kahn, R.S.," Automated separation of gray and white matter from MR images of the human Brain", NeuroImage 13, 230-237, 2001.

[4] Chalana, V., Ng, L., Rystrom, L.R., Gee, J.C., Haynor, D.R.," Validation of brain segmentation and tissue classification algorithm for T1-weighted MR images",. Med. Imag. 2001: Image Process. 4322, 1873-1882, 2001.

[5] Amato, U., Larobina, M., Antoniadis, A., Alfano, B.,"Segmentation of magnetic Resonance brain images through discriminant analysis", J. Neurosci. Methods 131,65-74, 2003.

[6] Zhu, C., Jiang, T.,"Multicontext fuzzy clustering for separation of brain tissues in magnetic resonance images", NeuroImage 18 (3), 685-696, 2003.

[7] ChihT.,Jim Z., MU-Der J., “A Fuzzy K-means clustering algorithm using cluster center displacement", journal of information science and engineering,vol. 27, 995-1009, 2011.

[8] Angela G. E. M. de Boer, T. T. Taskila, A. O. , Frank,Jos H. A., " Cancer survivors and Unemployment". The Journal of the American medical association JAMA, 30, 7,2009. 
[9] Gardner MJ. and DG. Altman, "Calculating confidence intervals for proportions and theirdifferences". BMJ Publishing Group, 28- 33,1989.

[10] Rai,G.N.Harikrishna, Nair, T.R. Gopalakrishnan "Gradient based seeded region grow method for CT angiographic image segmentation", In Proceedings of CoRR. 2010.

[11] Fan, J., Zeng, G., Body, M. ,Hacid, M., "Seeded region growing: an extensive andcomparative Study".Pattern Recognition Letters.26, 11391156, 2005.

[12] Jaccard P., "The distribution of the flora in the alpine zone". New phytol.11 (2),37 50,1912.

[13] Dice L., "Measures of the amount of ecologic association between species", Ecology,26,:297-302,1945.

[14] Brain Web, "Simulated Brain Database", McConnell Brain Imaging Centre, MontrealNeurological Institute, McGill.

[15] Zanaty E. A., S. Aljahdali "Fuzzy algorithms for automatic magnetic resonance image segmentation", International Arab Journal of Information Technology (IAJIT), 7, 3, 271-279, 2009.

[16] Zanaty E.A., S. Aljahdali, N. Debnath, "Improving fuzzy algorithms for automatic magnetic resonance image segmentation", Proceedings of seventeenth International Conference of Software Engineering and Data Engineering, 60-66, Los Angeles, California, USA, June 2008.
[17] Zanaty E.A., S. Aljahdali, N. Debnath, "A kernelized fuzzy c-means algorithm for Automatic magnetic resonance image segmentation", Journal of Computational Methods in Science and engineering (JCMSE), 123-136, 2009.

[18] Del-Fresno M., M. Vénere, and A. Clausse, A combined region growing and deformablemodel method for extraction of closed surfaces in 3D CT and MRI scans, ComputerizMedical Imaging and Graphics, 33, 369-376, 2009

[19] Yu Z. Q., Y. Zhu, J. Yang, Y. M. Zhu, A hybrid region-boundary model for cerebralcortical segmentation in MRI, Computerized Medical Imaging and Graphics, 30, 197208, 2006.

\section{AUTHOR'S PROFILE}

Ashraf Afifi is an assistance professor, faculty of computers and information technology, Taif University, Saudi Arabia. He received his MSC Degree in digital communication in 1995 from zagazig University, Egypt. He completed his $\mathrm{Ph}$. D. studies in 2002 from zagzig University,Egypt. His research interests are digital communication, and image segmentation. In these areas he has published several technical papers in refereed international journals or Conference proceedings. 